\title{
Usefulness of surveillance Cultures for Carbapenem- resistant Enterobacteriaceae, Carbapenem-resistant Pseudomonas aeruginosa and Vancomycin-resistant Enterococci in Hematopoietic Stem Cell Transplant Unit
}

Elisa Teixeira Mendes ( $\nabla$ elisatmendes@gmail.com )

Medical School/Pontifica Universidade Católica de Camnpinas https://orcid.org/0000-0003-4251-8185

Matias Chiarastelli Salomão

University of Sao Paulo: Universidade de Sao Paulo

Lísia Moura Tomichi

Hospital for Tropical Diseases

Maura Salaroli Oliveira

University of Sao Paulo: Universidade de Sao Paulo

Mariana Graça

Universidade de São Paulo Faculdade de Medicina: Universidade de Sao Paulo Faculdade de Medicina

Flavia Rossi

Universidade de Lisboa Instituto de Medicina Molecular: Universidade de Lisboa Instituto de Medicina Molecular Joao Lobo Antunes

Fernanda Spadão

Universidade de São Paulo Faculdade de Medicina: Universidade de Sao Paulo Faculdade de Medicina

Thais Guimaraes

Universidade de São Paulo Faculdade de Medicina: Universidade de Sao Paulo Faculdade de Medicina

Vanderson Rocha

universidade de sao paulo

Silvia Figueiredo Costa

Universidade de São Paulo

\section{Research}

Keywords: Hematopoietic Stem Cell Transplant infection, Hospital-acquired infection, Multidrug-resistant organisms, Surveillance Cultures, Blood Stream Infection

Posted Date: November 18th, 2020

DOI: https://doi.org/10.21203/rs.3.rs-109628/v1

License: (c) (i) This work is licensed under a Creative Commons Attribution 4.0 International License. Read Full License 


\section{Abstract}

Surveillance strategies to detect colonization is an important tool to prevent and control the spread of microorganisms especially among Hematopoietic Stem Cell Transplant (HSCT) patients. Colonization by Multidrug-resistant organisms (MDRO) has been evaluated as a risk factor for blood stream infection (BSI) in HSCT patients. The aim of this study was to evaluate the use of routine surveillance culture to screening colonization and infection by carbapenem-resistant Enterobacteriaceae (CRE), carbapenem-resistant Pseudomonas aeruginosa (CRPa) and vancomycin-resistant enterococci (VRE) in a HSCT unit.

Methods Surveillance cultures were collected from patients admitted to the HSCT unit over one-year, with swabs for cultures on admission and then weekly until discharge. We compared surveillance culture positivity for each site and agent, also clinical and epidemiological data according to the colonization status.

Results 200 HSCT patients underwent surveillance, with 1.323 samples collected. Infection due to MDRO occurred in $52(21.5 \%)$ patients, among them 45 (86.5\%) were blood stream infection (BSI) and 12 (23\%) had positive surveillance culture before infection. 554 (41.8\%) surveillance cultures were performed for CRPa, 413 (31.2\%) for VRE, and $356(27 \%)$ for CRE. Of these, 179 (13.5\%) surveillance culture were positive, with greater positivity for oropharynx $(6,35.3 \%) \mathrm{CRPa}$, and rectal samples $(16,20.7 \%)$ for CRE. Being colonized by any MDRO, CRE ( $p$ $<0.001)$ and CRPa $(p=0.027)$ was associated with a higher risk of infection in the bivariate analysis but being colonized was not associated with risk of death.

Conclusion Previous colonization by MDRO was a significant risk factor for infection by these pathogens, mainly colonization by CRE. Overall, rectal swab was the best site with the higher positivity, and the oropharynx was also an option for CRPa investigation. Feces culture showed low positivity and should be avoided. Although the impact of the strategy on the mortality of patients undergoing HSCT is not clear, VRE surveillance should be questioned in auto-HSCT patients as it has an additional cost and little impact on survival.

\section{Background}

Infections are the major cause of death in Hematopoietic Stem Cell Transplant (HSCT) patients [1]. These patients are at high-risk for acquiring health care infections and the use of antibiotics during febrile neutropenia leads to a higher prevalence of multi drug resistant organisms (MDRO) in this population, [2] in addition to the risk of dissemination in the transplant unit.

A previous study in our hospital has identified previous gut colonization by MDRO, particularly by Gram Negative bacteria, as associated with blood stream infection (BSI) in patients undergoing HSCT [3].

Surveillance strategies to detect colonization have been considered important tools for preventing and controlling the spread of MDRO in the hospital setting [4,5]. However, the cost effectiveness of this strategy in HSCT units and its impacts in patient's outcome is still controversy [6-8].

The aim of this study was to evaluate the use of routine surveillance culture to track colonization and infection by carbapenem-resistant Enterobacteriaceae (CRE), carbapenem-resistant Pseudomonas aeruginosa (CRPa) and vancomycin-resistant enterococci (VRE) in a HSCT unit.

\section{Methods}


The Hospital das Clínicas of Faculdade de Medicina da Universidade de São Paulo (HCFMUSP) is a tertiary hospital, in São Paulo, Brazil, with 2,200 beds. The HSCT unit is a 23-bed unit with 4 beds designated to allogeneic and 18 to autologous transplant. The beds designated for the allogeneic transplant are individual bedrooms, with positive pressure air circulation with high-efficiency particle air filter, and the beds designated for the autologous transplant are shared bedrooms, with the maximum number of two beds per room.

Antibacterial prophylaxis was administered with levofloxacin on the first day of stem cell infusion and discontinued when recovery of neutropenia or if the patients developed febrile neutropenia. Antiviral prophylaxis, antifungal and anti-Pneumocystis were administered according to guidelines [1].

Surveillance cultures were collected from patients admitted to the HSCT unit over one-year (2012). Swabs were collected for cultures on admission and then weekly until discharge, from multiple sites: axilla, feces, oropharynx and/or rectum.

The culture swabs for CRE and CRPa were incubated overnight in liquid media and then plated in Agar MacConkey medium with a meropenem disk (Ferreira et al 2018), and surveillance cultures of feces samples for VRE, in medium with vancomycin $6 \mathrm{mg} / \mathrm{L}$ [9].

Colonization was defined as the presence of at least one positive surveillance culture for one of the studied microorganisms.

Identification of resistant bacteria was performed with VITEK 2 (Biomeurieaux, Marcy-l'Étoile, France).

Clinical and epidemiological data were collected regarding sex, age, length of stay in BTM unit, diagnosis, use of antibiotics, infection, blood stream infection (BSI), and intra-hospital death. Infection was defined as CDC guidelines [10].

\section{Statistical analysis}

All data was stored in a database in Excel 97-2004 (Microsoft, Redmond, WA, United States). We compared surveillance culture positivity for each site and agent. Clinical and epidemiological data were analyzed according to the colonization status. All statistical analyses were performed using Epilnfo 7.0 (CDC, Atlanta, United States) Fisher's exact test or Chi-square test were used for categorical variables, as appropriate, and Mann-Whitney and Log-Rank testes for continuous variable. Univariate analysis and multivariate logistic regression analysis were performed (95\% confidence interval). We considered a $P$ value $<0.05$ as statistically significant. A Kaplan-Meier curve was generated to compare survival among patients with and without BSI.

\section{Results}

A total of 200 HSCT patients underwent surveillance, with 1.323 samples collected. The mean age was 45 years, 107 (53.5\%) males and $17.7 \%$ of hospital death, and 59\% patients performed Auto-transplantation as shown in Table 1. 
Table 1

Characteristic of patients who underwent a surveillance culture, in HSCT ward, Hospital of Clinics, São Paulo

\begin{tabular}{|c|c|}
\hline & $\mathbf{N}(\%)$ \\
\hline Total & $200(100)$ \\
\hline Age mean (years) & 45 y \\
\hline Sex (Male) & $107(53.5)$ \\
\hline Surveillance samples & $1323(100)$ \\
\hline Allo-HSCT & $82(41)$ \\
\hline LOS in days. Mean (range) & $19.4(1-66)$ \\
\hline LOS in days until MDRO colonization. Mean (range) & $16(0-55)$ \\
\hline CRE & $18(1-55)$ \\
\hline CRPa & $20(0-39)$ \\
\hline VRE & $10.5(0-40)$ \\
\hline LOS in days until MDRO infection & $23(0-77)$ \\
\hline Mean (range) & $26.7(0-77)$ \\
\hline CRE & $21(0-51)$ \\
\hline CRPa & 19 \\
\hline \multicolumn{2}{|l|}{ VRE* } \\
\hline Hospital Death & $43(17.7)$ \\
\hline $\begin{array}{l}\text { MDR- Multi-Drug Resistant Organism, Allo- allogenic, } \\
\text { Carbapenem Resistant Pseudomonas aeruginosa; V } \\
\text { Stay. *Only one case of VRE infection }\end{array}$ & $\begin{array}{l}\text { robacteriaceae, CRPa } \\
\text { occi. LOS- Length of }\end{array}$ \\
\hline
\end{tabular}

We performed 554 (41.8\%) surveillance cultures were performed for CRPa, 413 (31.2\%) for VRE, and 356 (27\%) for CRE. Of these, 179 (13.5\%) surveillance culture were positive, with greater positivity for oropharynx $(6,35.3 \%)$ CRPa, and rectal samples $(16,20.7 \%)$ for CRE. Feces samples performed displayed only $12.6 \%$ of positivity, and CRE positivity was two times higher in rectal cultures. Infection due to MDRO occurred in 52 (21.5\%) patients, among them 45 (86.5\%) were bacteremia and 12 (23\%) had positive surveillance culture before infection. Median detection time to positivity surveillance cultures until infection due to MDRO was 21.4 days for infection due CRE and 14.1 days for infection due CRPa (Table 2). 
Table 2

Data from surveillance cultures, collected from inpatients at the HSCT ward, Hospital of Clinics, São Paulo

\begin{tabular}{|lllllll|}
\hline $\begin{array}{l}\text { Survillance } \\
\text { culture }\end{array}$ & Total (\%) & $\begin{array}{l}\text { Positive } \\
\text { Samples }\end{array}$ & \multicolumn{2}{l}{ Positivity by site } & \\
\cline { 5 - 7 } & & & $\begin{array}{l}\text { Axilary } \\
(116)\end{array}$ & $\begin{array}{l}\text { Feces } \\
(1109)\end{array}$ & $\begin{array}{l}\text { Rectal } \\
\text { Oropharynx }\end{array}$ & (17) \\
\hline Total Samples & 1324 & $179(13.5)$ & $16(13.8)$ & $140(12.6)$ & 17 & $6(35.3)$ \\
CRE (\%) & $(100)$ & $85(23.8)$ & $62(20.7)$ & $62(20.7)$ & 13 & $5(45.5)$ \\
CRPa (\%) & $356(27)$ & $41(7.4)$ & $28(6.9)$ & $28(6.9)$ & $(39.4)$ & $1(14.3)$ \\
VRE (\%) & $554(41.8)$ & $53(12.8)$ & $50(12.5)$ & $50(12.5)$ & $1(2.6)$ & $3(25)$ \\
& $413(31.2)$ & & & & $3(25)$ & \\
\hline
\end{tabular}

CRE - Carbapenem Resistant Enterobacteriaceae, CRPa - Carbapenem Resistant Pseudomonas aeruginosa; VRE Vancomycin Resistant Enterococci

The average LOS time for colonization and MDRO infection was 16 and 23 days respectively. Infection and colonization by VRE occurred earlier, when compared to other bacteria, however having VRE was not a risk factor for MDRO infection and death

Being colonized by any MDRO, CRE $(p<0.001)$ and CRPa $(p=0.027)$ was associated with a higher risk of infection in the bivariate analysis, and only colonization by $\operatorname{CRE}(p=0.009)$ remained significant in the multivariate analysis (Table 3).

Risk for death was significantly higher among patients with any MDRO infection, including CRE and CRPa in the bivariate analysis $(p<0.001)$, whereas in the multivariate analysis, only CRPa infection $(p=0.028)$ remained significant (Tables 3 and 4).

Table 3.

Risk factors for death and infection - total and stratified by each agent in a bivariate analysis, in a HSCT ward, Hospital of Clinics, São Paulo 
th

s factor Deaths/Patients Deaths/Patients

\begin{tabular}{|c|c|c|c|}
\hline with risk factor & without risk & OR & CI (95\%) \\
\hline (\%) & 10 & & \\
\hline
\end{tabular}

snization
20/94 (21.28)
$22 / 145$ (15.17)
1. 08
0.95
1.22
0. 22

any

RO

$\begin{array}{lcccccc}\text { CRE } & 12 / 50(24) & 30 / 189(15.87) & 1.11 & 0.94 & 1.31 & 0.18 \\ \text { CRPa } & 5 / 31(16.13) & 37 / 208(17.79) & 0.98 & 0.83 & 1.16 & 0.82 \\ \text { VRE } & 7 / 47(14.89) & 35 / 192(18.23) & 0.96 & 0.84 & 1.10 & 0.59 \\ \text { ction } & & & & & \\ & 22 / 44(50) & 21 / 196(10.71) & 1.79 & 1.32 & 2.41 & <0.001\end{array}$

any

RO

$\begin{array}{lllllll}\mathrm{CRE} & 10 / 21(47.62) & 33 / 219(15.07) & 1.62 & 1.07 & 2.44 & <0.001\end{array}$

$\begin{array}{lllllll}\mathrm{CRPa} & 16 / 26(61.54) & 27 / 214(12.62) & 2.27 & 1.39 & 3.70 & <0.001\end{array}$

VRE $\quad 1 / 1(100)$

$42 / 239$ (17.57) Undefined Undefined Undefined 0.18

\section{Infection}

: factor Infections/Pat. Infections /Pat.

$\begin{array}{ccccc}\text { with risk factor } & \text { without risk } & \text { OR } & \text { CI (95\%) } & \text { P } \\ (\%) & \text { factor (\%) } & & \end{array}$

snization
26/94 (27.66\%)
$17 / 145$ (11.72\%)
1.22
1.06
1.40
0.002

any

RO

\begin{tabular}{lcccccc} 
CRE & $19 / 50(38 \%)$ & $24 / 189(12.70 \%)$ & 1.41 & 1.13 & 1.76 & $<0.001$ \\
CRPa & $10 / 31(32.26 \%)$ & $33 / 208(15.87 \%)$ & 1.24 & 0.97 & 1.59 & 0.027 \\
VRE & $12 / 47(25.53 \%)$ & $31 / 192(16.15 \%)$ & 1.13 & 0.94 & 1.35 & 0.133 \\
\hline
\end{tabular}

Infection by Carbapenem Resistant Enterobacteriaceae

Infection Infection CRE/Pat.

s factor

CRE/Pat. with without risk

OR

CI (95\%)

$\mathbf{P}$ 
risk factor (\%) factor(\%)

mization by:

\begin{tabular}{lcccccc} 
any MDRO & $13 / 94(13.83 \%)$ & $8 / 145(5.52 \%)$ & 1.10 & 1.002 & 1.20 & 0.027 \\
CRE & $10 / 50(20 \%)$ & $11 / 189(5.82 \%)$ & 1.18 & 1.02 & 1.36 & 0.004 \\
CRPa & $2 / 31(6.45 \%)$ & $19 / 208(9.13 \%)$ & 0.97 & 0.88 & 1.07 & 1.00 \\
VRE & $5 / 47(10.64 \%)$ & $16 / 192(8.33 \%)$ & 1.03 & 0.92 & 1.14 & 0.57 \\
\hline
\end{tabular}

\section{Infection by Carbapenem Resistant Pseudomonas aeruginosa}

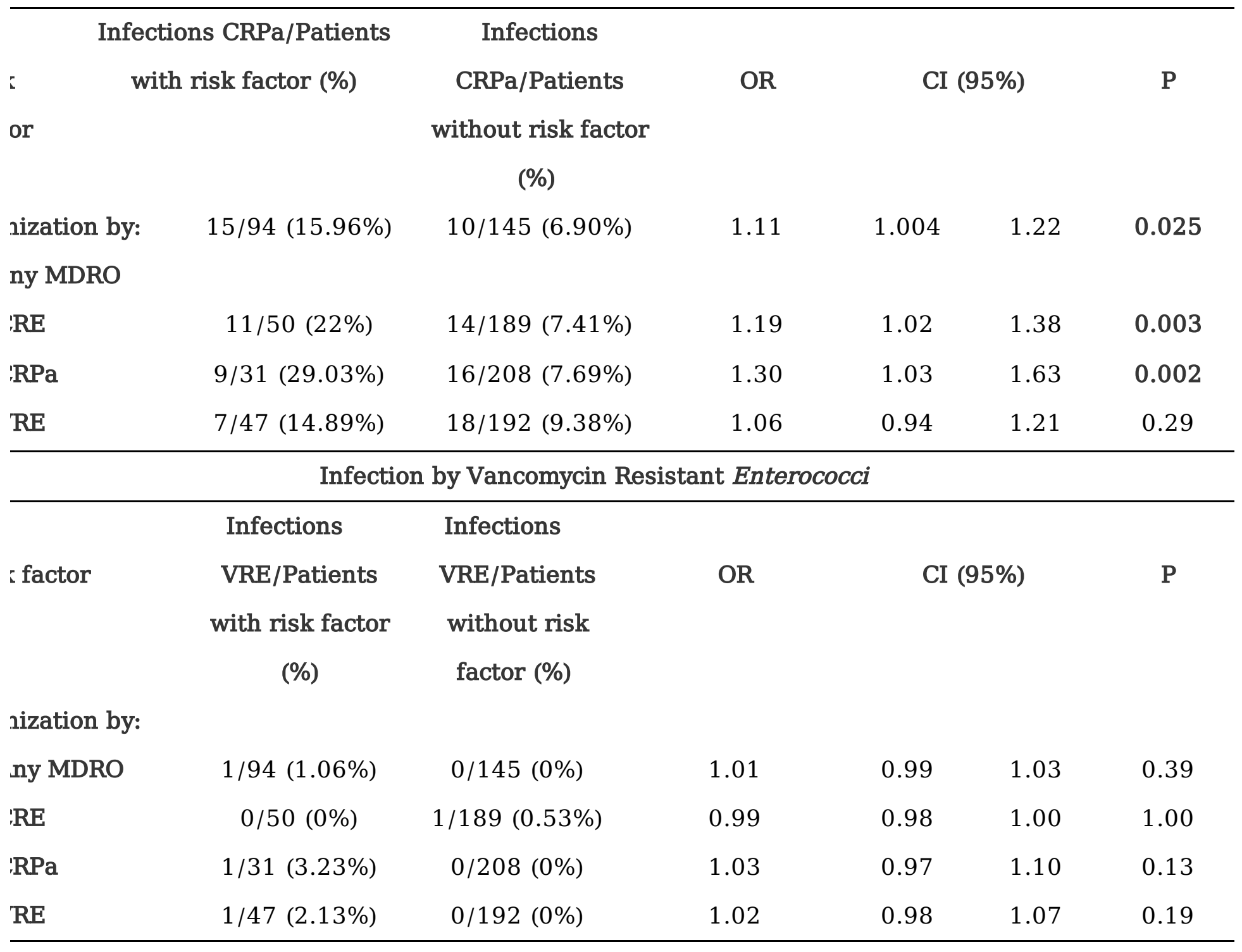

CI - Confidence Interval; MDRO - Multi-Drug Resistant Organism (CRE. CRPa or VRE); CRE Carbapenem Resistant Enterobacteriaceae; CRPa - Carbapenem Resistant Pseudomonas aeruginosa; VRE - Vancomycin Resistant Enterococci. 
Table 4.

Variables associated with infection in a multiple logistic regression, BTM Unit, Hospital of Clinics, São

\begin{tabular}{|lllll|}
\hline \multicolumn{5}{c}{ Paulo } \\
\hline Infection & & & & \\
\hline Colonization by: & OR & \multicolumn{2}{c|}{ Cl (95\%) } & P \\
CRE & 4.54 & 1.46 & 14.13 & $\mathbf{0 . 0 0 9}$ \\
CRPa & 1.91 & 0.68 & 5.35 & 0.220 \\
VRE & 1.77 & 0.60 & 5.23 & 0.304 \\
any MDRO & 0.71 & 0.18 & 2.89 & 0.636 \\
\hline
\end{tabular}

OR - Odds Ratio; Cl - Confidence Interval; MDRO - Multi-Drug Resistant Organism (CRE. CRPa or VRE); CRE Carbapenem Resistant Enterobacteriaceae, CRPa - Carbapenem Resistant Pseudomonas aeruginosa; VRE Vancomycin Resistant Enterococci.

CRE infection occurred more significantly among CRE colonized ( $p=0.004)$, and CRPa infection occurred more among CRPa colonized $(p=0.002)$. Having any MDRO was associated with a higher risk of infection in a bivariate analysis (Table 2).

Chance of Survival was significantly lower among patients with BSI, as demonstrated in the Kaplan Meier curve (P $=0.012)$, as well as among those infected with CRPa (0.0053).

\section{Discussion}

The prevalence of colonization and type MDRO varies greatly according to the study centers, depending on epidemiology and local practices, such as the use of antibiotic prophylaxis, type of HSCT, among other factors [58]. In this study, the positivity of the surveillance culture was $13.5 \%$ lower than data from Europe [4, 11]. CRPa and VRE were the main MDRO, both colonizing $42 \%$ of patients with MDRO. The average length of hospital stays until the first positive culture varied according to the pathogen, the time for colonization with VRE was 10 days while for CRPa was much longer (20 days). Heidenreich et al diagnosed 27\% of colonization by MDRO in the first 100 days after transplantation, with a predominance of CRPa (26.9\%), with a pre-induction prevalence of $16 \%$ in German (11). Sadowska-Klasa in Poland obtained $42 \%$ colonization by MDRO during hospitalization, with an important predominance of VRE [4].

In this cohort, previous colonization by MDRO was a significant risk factor for infection by these pathogens, mainly colonization by CRE. A study at our center, in the 2014-2015 period, also reported that colonization by previous MDRO was associated with BSI ( $<<0.001)$, with $20 \%$ of patients colonized by GNB-MDR developed BSI by these agents [3]. Other studies have also found similar findings $[12,13]$ Therefore, strategies for selective decolonization of the gastrointestinal tract (SDD) have been evaluated. A single center study demonstrated costeffectiveness of SDD in CRE colonized patients in intensive care units [14]. However, a systematic review of 2019 
still classifies the evidence indicating benefit in decolonization as limited and does not recommend this routine intervention, moreover. studies with immunocompromised patients are still extremely scarce in the literature [15].

Although, colonization is associated with a higher risk of infection, the impact of the strategy on the mortality of patients undergoing HSCT is not clear. We observed that infection by any MDRO, CRE and mainly by CRPa was associated with the risk of death; moreover, being colonized was not a risk factor for death. In other studies, a higher risk of death was observed in colonized patients. Sadowska-Klasa et al. [4] and Bilinski et al. [7] evaluated patients undergoing allogeneic HSCT and in both studies, colonization by MDRO had an impact on overall survival (OS) in 1 year. The relative high rate of Auto-HSCT in our series (59\%) may explain our results.

In our study, we obtained a high prevalence of VRE colonization; which was the only MDRO that did not affect the risk of infection. Perhaps its lower virulence in relation to gram-negative bacillus (GNB) has influenced the lower impact of colonization by MDRO on hospital mortality in our population [16]. In contrast, a study conducted at the Mayo Clinic, with a 10-year series evaluated the influence of colonization by VRE on the prognosis of patients undergoing Allo-HSTC by AML. In multivariate analysis, colonization by VRE was an independent risk factor for VRE infection, but did not influence any other post-transplant outcome [8].

Corroborating this point, in a multicenter study carried out in Italy ( 52 centers), being colonized by resistant gramnegative bacteria significantly reduced 4-month survival. In addition, colonization by CRE and CRPa increased the risk of infection with these pathogens $(p<0.001)$ [16]. On the other hand, Heidenreich and colleagues [11] also found a similar risk of death regardless of the status of colonization by MDRO, even though CRE was the main colonizer in his studied population [11].

Our data corroborate the fact that the rectal swab is more sensitive than the culture of feces $[18,19]$, especially regarding VRE and CRE. Despite this, the use of rectal swab in HSCT patients should be used with care, to avoid skin or mucosal breakdown during severe neutropenia. In this scenario, the oropharyngeal swab may be an alternative, since it presented a high positivity for CRE and VRE. We observed that the type of surveillance culture site depends on the pathogen, feces culture showed low positivity and should be avoided; and VRE surveillance should be questioned in patients undergoing autologous transplantation because it has an additional cost and little impact on survival and development of bloodstream infection.

The present study, in addition to being carried out in a single center, has the disadvantage of being retrospective. However, it brings important reflections to the practice of screening MDR in TCTH patients. Firstly, being colonized by MDRO does not seem to be a sufficient factor to interfere in post-HSCT survival, especially VRE colonization and in auto-HSCT patients.

\section{List Of Abbreviations}

BSI - blood stream infection

CRE - carbapenem-resistant Enterobacteriaceae

$\mathrm{Cl}$ - Confidence Interval

CRPa - carbapenem-resistant Pseudomonas aeruginosa

GNB - Gram-negative bacteria

Page 9/13 
HSCT - Hematopoietic Stem Cell Transplant

LOS - Length of stay

MDRO - Multi-drug-resistant organisms

VRE - vancomycin-resistant enterococci

\section{Declarations}

\section{Ethics approval}

This study was evaluated and approved by the ethics committee of the Hospital of Clinics, São Paulo University. Protocol number: CAAE: 50237715.4.0000.0068

\section{Consent for publication}

All authors cited consented to the publication

\section{Availability of data and materials}

The datasets analyzed during the current study are available from the corresponding author on reasonable request.

\section{Competing interests}

There are no conflicts of interest involved

\section{Funding}

No funds were used in this paper

\section{Authors' contributions}

Elisa Teixeira Mendes: Literature review, statistical analysis and paper writing

Matias Salomão, Lísia Moura Tomishi, Maura Salaroli Oliveira, Fernanda Sapadão, Thais Guimarães, Vanderson Rocha: Data collection, database construction, literature review, statistical analysis

Mariana Graça, Flavia Rossi: Laboratory and microbiological work and analysis

Silvia Figueiredo Costa: study design, orientation and structuring of the research and review the manuscript.

\section{Acknowledgements:}

We thank our HSCT patients

\section{References}


1. Tomblyn $\mathrm{M}$, Chiller $\mathrm{T}$, Einsele $\mathrm{H}$, et al. Guidelines for preventing infectious complications among hematopoietic cell transplantation recipients: a global perspective. Biol Blood Marrow Transplant. 2009;15(10):1143-238.

2. Averbuch D, Tridello G, Hoek J, Mikulska M, Akan H, Yaňez L, et al. Antimicrobial Resistance in Gram-Negative Rods Causing Bacteremia in Hematopoietic Stem Cell Transplant Recipients: Intercontinental Prospective Study of the Infectious Diseases Working Party of the European Bone Marrow Transplantation Group. Clinical Infectious Diseases. 2017;65(11):1819-28

3. Ferreira AM., Moreira F, Guimaraes T, Spadão F, Ramos J F, Batista MV, Rocha V. Epidemiology, risk factors and outcomes of multi-drug-resistant bloodstream infections in hematopoietic stem cell transplant recipients: importance of previous gut colonization. Journal of Hospital Infection.2018;100(1), 83-91.

4. Sadowska-Klasa A, Piekarska A, Prejzner W, Bieniaszewska M, Hellmann A. Colonization with multidrugresistant bacteria increases the risk of complications and a fatal outcome after allogeneic hematopoietic cell transplantation. Annals of Hematology. 2018; 97:509-517

5. Patriarca F, Cigana C, Massimo D, Lazzarotto D, Geromin A, Isola M, Candoni A. Risk factors and outcomes of infections by multidrug-resistant gram-negative bacteria in patients undergoing hematopoietic stem cell transplantation. Biology of Blood and Marrow Transplantation. 2017; 23(2):333-339

6. Forcina A, Lorentino F, Marasco V, Oltolini C, Marcatti M, Greco R, et al. Clinical Impact of Pretransplant Multidrug-Resistant Gram-Negative Colonization in Autologous and Allogeneic Hematopoietic Stem Cell Transplantation. Biol Blood Marrow Transplant. 2018;24:1476-1482

7. Bilinski J, Robak K, Peric Z, Marchel H, Karakulska-Prystupiuk E, Halaburda K, et. Al. Impact of Gut Colonization by Antibiotic-Resistant Bacteria on the Outcomes of Allogeneic Hematopoietic Stem Cell Transplantation: A Retrospective, Single-Center Study. Biol Blood Marrow Transplant. 2016;22(6):1087-1093.

8. Hefazi M, Damlaj M, Alkhateeb HB, Partain DK, Patel R, Razonable RR, et al. Vancomycin-resistant Enterococcus colonization and bloodstream infection: prevalence, risk factors, and the impact on early outcomes after allogeneic hematopoietic cell transplantation in patients with acute myeloid leukemia. Transpl Infect Dis. 2016;18(6):913-920.

9. Lisboa LF, Miranda BG, Vieira MB, Dulley FL, Fonseca GG, Guimarães T, Levin AS, Shikanai-Yasuda MA, Costa SF. Empiric use of linezolid in febrile hematology and hematopoietic stem cell transplantation patients colonized with vancomycin-resistant Enterococcus spp. Int J Infect Dis. 2015;33:171-6.

10. O'Grady NP, Alexander M, Burns LA, Dellinger P, Garland J, Heard SO. Guidelines for the Prevention of Intravascular Catheter-Related Infections, 2011. https://www.cdc.gov/infectioncontrol/guidelines/bsi/index.html

11. Heidenreich D, Kreil S, Jawhar M, Müller N, Nolte F, Becker KP, et al. Course of colonization by multidrugresistant organisms after allogeneic hematopoietic cell transplantation. Annals of Hematology. 2018; 97:2501-2508

12. Nesher L, Rolston KVI., Shah DP, Tarrand JT, Mulanovich V, Ariza-Heredia EJ, Chemaly RF. Fecal colonization and infection with Pseudomonas aeruginosa in recipients of allogeneic hematopoietic stem cell transplantation. Transpl Infect Dis. 2015;17:33- 38

13. Demiraslan H, Cevahir F, Berk E, Metan M, Cetin M, Alp DE, et al. Is surveillance for colonization of carbapenem-resistant gram-negative bacteria important in adult bone marrow transplantation units? American Journal of Infection Control. 2017; 45(7):735-739. 
14. You JH, Li HK, Ip M. (Surveillance-guided selective digestive decontamination of carbapenem-resistant Enterobacteriaceae in the intensive care unit: A cost-effectiveness analysis. American Journal of Infection Control, 2018;46(3), 291-296.

15. Tacconelli E, Mazzaferri F, Smet AM, Bragantini D, Eggimann P Huttner BD, et al. ESCMID-EUCIC clinical guidelines on decolonization of multidrug-resistant Gram-negative bacteria carriers. Clin Microbiol Infect. 2019;25(7):807-817

16. Prado GVB, Marchi AP, Moreno LZ, Rizek C, Amigo U, Moreno AM, Rossi F, Guimaraes T, Levin AS, Costa SF. Virulence and resistance pattern of a novel sequence type of linezolid-resistant Enterococcus faecium identified by whole-genome sequencing. J Glob Antimicrob Resist. 2016;6:27-31

17. Girmenia C, Bertaina A, Piciocchi A, et al. Incidence, risk factors and outcome of pre-engraftment gramnegative bacteremia after allogeneic and autologous hematopoietic stem cell transplantation: an Italian prospective multicenter survey. Clin Infect Dis. 2017;65(11):1884-1896.

18. Aschbacher R, Pagani L, Migliavacca R, Pagani L; GLISTer (Gruppo di Lavoro per lo Studio delle Infezioni nelle Residenze Sanitarie Assistite e Strutture Assimilabili) working group. Recommendations for the surveillance of multidrug-resistant bacteria in Italian long-term care facilities by the GLISTer working group of the Italian Association of Clinical Microbiologists (AMCLI). Antimicrob Resist Infect Control. 2020;13:9(1):106.

19. Yan L, Sun J, Xu X, Huang S. Epidemiology and risk factors of rectal colonization of carbapenemaseproducing Enterobacteriaceae among high-risk patients from ICU and HSCT wards in a university hospital. Antimicrob Resist Infect Control. 2020; 23;9(1):155. doi: 10.1186/s13756-020-00816-4. PMID: 3296771

\section{Figures}

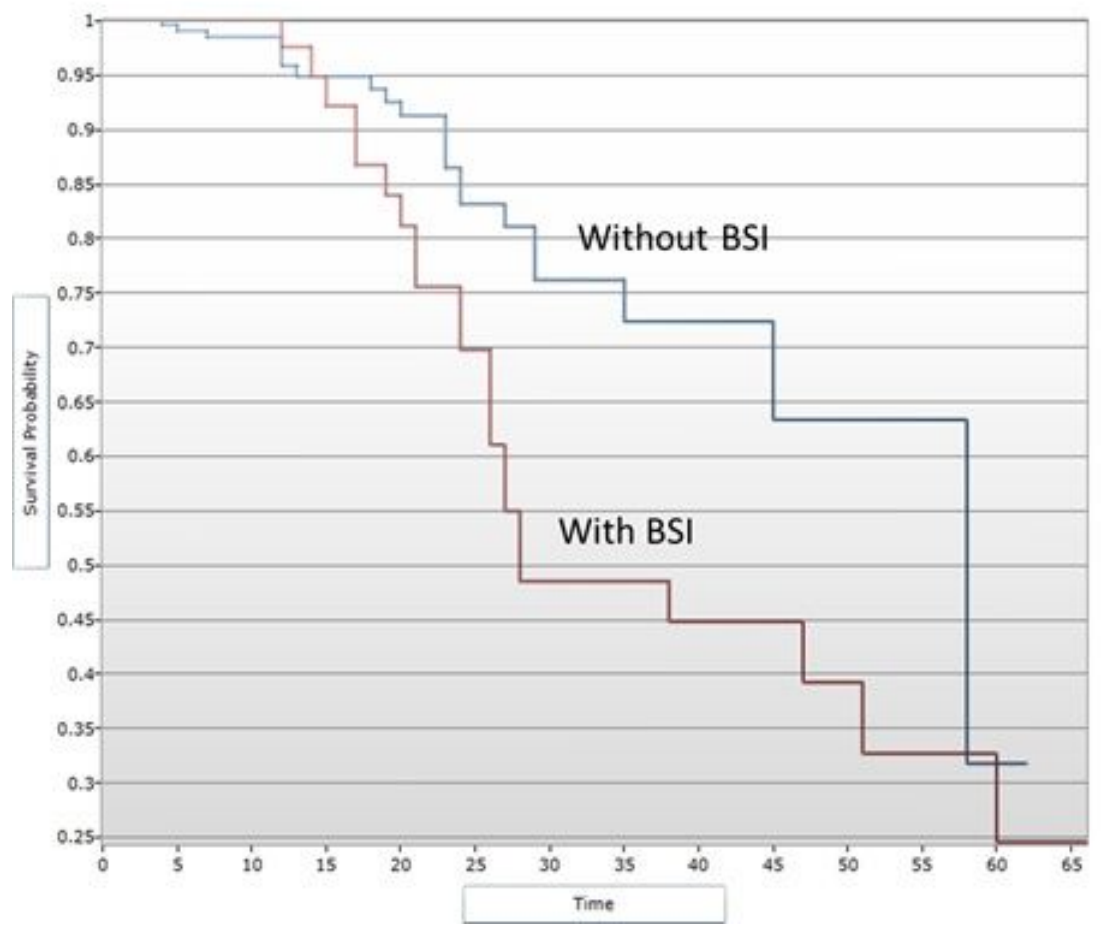

Figure 1 
Survival analysis by Kaplan Meier curve in patients undergoing HSCT with and without BSI - Hospital of Clinics, São Paulo

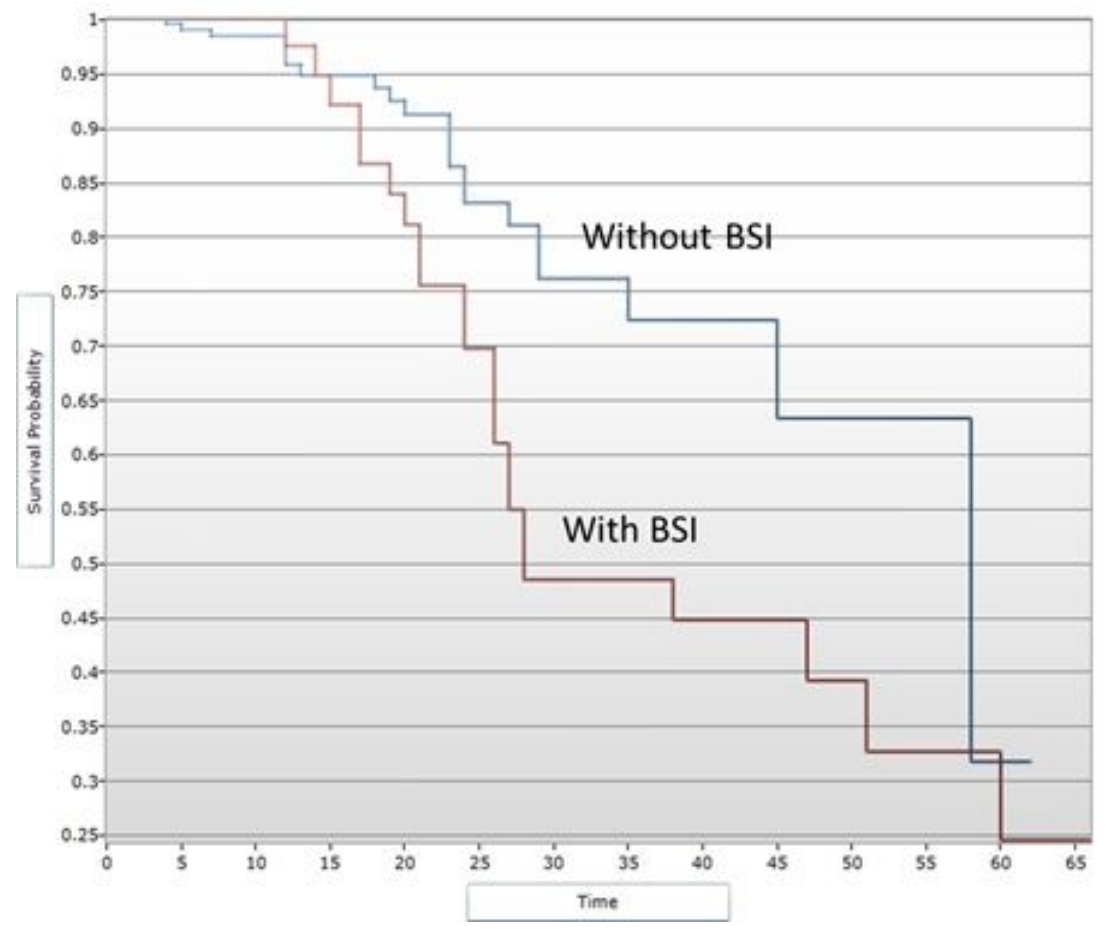

\section{Figure 1}

Survival analysis by Kaplan Meier curve in patients undergoing HSCT with and without BSI - Hospital of Clinics, São Paulo 